\title{
An Ontology Design Pattern for Referential Qualities
}

\author{
Jens Ortmann and Desiree Daniel \\ Institute for Geoinformatics, University of Muenster, Germany \\ jens.ortmann@uni-muenster.de, \\ desiree.daniel@yahoo.com
}

\begin{abstract}
Referential qualities are qualities of an entity taken with reference to another entity. For example the vulnerability of a coast to sea level rise. In contrast to most non-relational qualities which only depend on their host, referential qualities require a referent additional to their host, i.e. a quality $\mathrm{Q}$ of an entity $\mathrm{X}$ taken with reference to another entity R. These qualities occur frequently in ecological systems, which make concepts from these systems challenging to model in formal ontology. In this paper, we discuss exemplary resilience, vulnerability and affordance as qualities of an entity taken with reference to an external factor. We suggest an ontology design pattern for referential qualities. The design pattern is anchored in the foundational ontology DOLCE and evaluated using implementations for the notions affordance, resilience and vulnerability.
\end{abstract}

\section{Introduction}

Environmental problems are major challenges of the $21^{\text {st }}$ century and occur in all parts of the world on local, regional and global scales, especially in face of climate variability. The rate of global environmental change is surpassing response and without action to mitigate its drivers and enhance societal resilience, these changes will retard economic, social, environmental and developmental goals [16]. According to the International Council for Science [16], to meet this challenge, what is required is a robust information infrastructure that can combine data and knowledge both past and present with new observations and modeling techniques to provide integrated, interdisciplinary datasets and other information products. One key functionality within this infrastructure is to assess vulnerability and resilience.

Before such an infrastructure can be realized, there is a need to sort out the bottleneck that occurs on the conceptual level with notions such as vulnerability and resilience. In an attempt to introduce ecological concepts to formal ontology, the problem of modeling qualities arises. In addition to their host, qualities in the ecological domain often depend on external entities. A recurring pattern is $a$ quality $Q$ of an entity $X$ taken with reference to another entity $R$. These qualities have one host and cannot exist without an external factor. To name just one example, the vulnerability of a coast cannot be assessed per se, but only with respect to an external threat, like the vulnerability to sea level rise. So we have 
the vulnerability of a coast with reference to sea level rise. Yet, vulnerability is not considered a relational quality, but is attributed to the coast. Furthermore, the same coast has different vulnerabilities with reference to different external factors (e.g., a hurricane or an oil-spill).

Modeling qualities that inhere in more than one host represents a conceptual quandary that applies to ecological notions but it appears in many other fields that deal with systems (e.g. economic systems, social systems, communication networks). We take a look at the notions of resilience, vulnerability and affordance, which lack formal definitions. An approach that can model referential qualities in ontologies can lead to these concepts being implementable and thereby usable in semantic web applications.

This paper therefore brings into focus the research question "how can referential qualities be modeled in ontologies?". To solve the quandary, an Ontology Design Pattern (ODP) [8] is suggested as a template to model different referential qualities. This work draws from Kuhn's 20 21] Semantic Reference Systems. Semantic Reference Systems provide a theory of how categories in an ontology can be described with reference to other, already established categories. At the same time the theory of Semantic Reference Systems paves the road to make these ecological qualities operational. A high level formalization of the Ontology Design Pattern as well as an OWL-DI 11 implementation is carried out by the authors. Both are aligned to the Descriptive Ontology for Linguistic and Cognitive Engineering (DOLCE) 23. The ecological qualities resilience, vulnerability and affordance serve to illustrate applications of the suggested ODP.

This paper proceeds by outlining the background on non-relational qualities, relational qualities and Ontology Design Patterns in Sect. 2. Subsequently, Section 3 motivates this research by discussing three examples of referential qualities. Section 4 describes the Ontology Design Pattern for referential qualities and its implementation in OWL-DL. The implementation of the examples are discussed in Sect. 5. In Sect. 6 the conducted research is evaluated against the use cases as well as analytically. Finally, Sect. 7 concludes the paper.

\section{Background}

This section introduces the notions of non-relational qualities and relational qualities with respect to DOLCE as well as the idea of the ODP approach.

\subsection{Non-relational and Relational Qualities}

In philosophy there exist several dichotomies of qualities (or properties), they are labeled for example "intrinsic-extrinsic", "relational-non-relational", "unaryn-ary" (cf. eg., 38). The differences between these distinctions are sometimes hard to grasp for non-experts, as for instance many standard examples of nonrelational qualities are intrinsic and unary (e.g. color of a rose), and many

\footnotetext{
${ }^{1}$ The description logic (DL) variant of the web ontology language (OWL). See http://www.w3.org/TR/owl-guide/ for more information. All websites referred to in this paper were last accessed on 21.06.2011.
} 
standard examples of relational qualities are extrinsic and binary (e.g. gravitational attractions). However, there exist intrinsic relational qualities and extrinsic non-relational qualities. In the following we will introduce the relational non-relational distinction, as we perceive this one as the most intuitive to nonexperts in metaphysics. We only mention the intrinsic-extrinsic distinction as additional criterion when necessary.

Non-Relational Qualities. Non-relational qualities can be modeled in DOLCE. In essence, this can be seen as an ODP in itself, even though to our knowledge it is never officially declared as such. The design pattern for modeling qualities in DOLCE follows the EQQS (Endurant, Quality, Quale, Spaces)pattern described in 22. The pattern comprises four categories. An endurant2 (E) that serves as host for the quality $(\mathrm{Q})$, with a quale $(\mathrm{Q})$ that is located in a quality-space (S). In DOLCE all entities are particulars [23]. Every particular endurant has its own particular qualities. For example, we can say that two boys have the same height, however, in DOLCE each boy has his own distinct height quality. This allows qualities to change, for instance, if the boys grow, especially when they grow at different rates and do not have the same heights as adults any more. To make the distinct qualities operational, a quale is needed. The quale is a representation of the quality in a quality space. For example, in a meter space the height quality of the two boys is reflected by the same quale. The quale is modeled as a region in a quality-space, which allows certain computations, depending on the structure of the space. An illustration of the DOLCE quality ODP is shown in Fig. 1. The formal relation and implementation of the quality ODP can be found in [23].

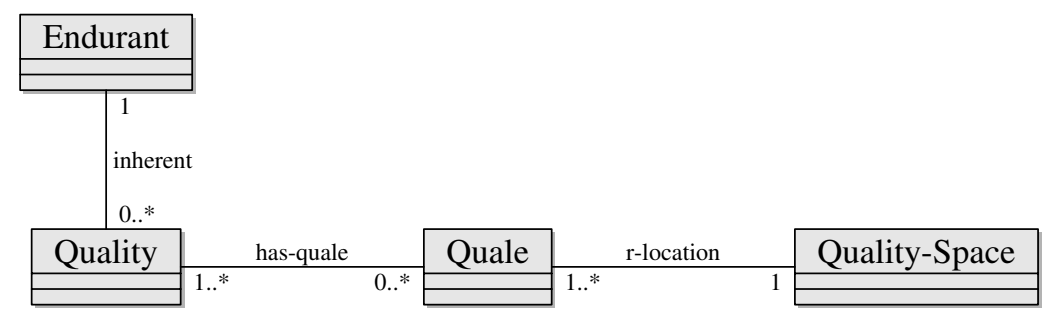

Fig. 1. UML class diagram of the quality ODP in DOLCE. A quality inheres in an endurant. The quality has a quale that is located in a quality space.

Relational Qualities. Relational qualities inhere in more than one host. This is not allowed in DOLCE, where the quality can only inhere in one host. For example the solubility of a substance in a liquid cannot be expressed in the EQQS pattern in DOLCE, because the solubility inheres in both, the substance and the

${ }^{2}$ Throughout the paper we use truetype fonts like quality to refer to categories in a formal ontology. 
liquid. DOLCE falls short modelling qualities like solubility, distance, direction [29]. Qualities that depend on two entities are often extrinsic and relational. They usually qualify a relation between two entities. The formal relation that expresses a relational quality has more than two entities, namely the quality and the hosts. Therefore, a direct implementation in Description Logics is not possible. However, there exists a logical ontology design pattern to transform n-ary relations expressed in a more expressive logic into OWL [26]. This pattern can be used to encode relational qualities as well. Nonetheless, this model diverts from the DOLCE EQQS pattern for qualities, and it violates DOLCE's constraint that a quality can only inhere in one host.

\subsection{Ontology Design Patterns}

Ontology Design Patterns are recurring structures in ontologies. Similar to design patterns in software engineering they provide templates to specific problems in engineering ontologies. The first patterns were not designed from scratch but emerged in ontologies. A first account of this phenomenon in ontology engineering along with a framework to document and classify ontology design patterns was given by Gangemi [8]. Gangemi and Presutti [10] list different types of ontology design patterns, depending on their intended use. They distinguish between Structural ODPs, Reasoning ODPs, Presentation ODPs, Correspondence ODPs, Lexico-Syntactic ODPs and Content ODPs for example. The details on these different ODPs can be found in [10] or looked up on the website ontologydesignpatterns.org. In this paper we are only interested in Content ODPs, and will refer to them as "ODP" for short. They can be defined as:

[Content ODPs] are distinguished ontologies. They address a specific set of competency questions, which represent the problem they provide a solution for. Furthermore, [Content ODPs] show certain characteristics, i.e. they are: computational, small, autonomous, hierarchical, cognitively relevant, linguistically relevant, and best practises. [10, p. 231]

Most important in this definition is that Content ODPs are small ontologies. Gangemi and Presutti [10] also suggest a new paradigm for ontology engineering, based on small modular Content ODPs, which form that backbone of a new ontology. The website ontologydesignpatterns.org is a community portal intended to serve as hub for finding and contributing ODPs of all types.

\section{$3 \quad$ Examples}

In this section, we discuss the salience of affordance, resilience and vulnerability as well as the problems that occur on the conceptual level when modeling these notions.. A major problem is still, that these qualities cannot be modelled in formal ontology. This hinders modelling ecological systems in information infrastructures. 


\subsection{Resilience}

The notion of resilience originated within the Ecology domain through the work of Holling [15. From its beginning in ecology, resilience has transcended across various disciplines such as Sustainable Science, Disaster Management, Climate Change and Psychology [13618,13] as it is deemed a salient concept pertaining to systems in face of adversity. Holling [15] ascertained that ecological resilience is a characteristic of ecosystems to maintain themselves in face of disturbance. In other words, resilience can be viewed as a quality of a system.

After four decades of the resilience debate, the concept still remains on the abstraction level. Instead of domains moving into an operational direction, there is a constant re-invention of the wheel that hinders research. This impediment can be attributed to the lack of a common lexicon across domains [24. Walker et al. 35] state that in order for resilience to be operational we have to consider resilience in a specific context 'from what to what', for example the resilience of corn in South Africa to the impacts of drought. This specificity brings into focus the problem of having a quality that is dependent on a referent and the limitations of DOLCE in this regard.

Since the inception of resilience in Ecology, several definitions across disciplines have emerged. Holling initially described resilience in contrast to stability:

Resilience determines the persistence of relationships within a system and is a measure of the ability of these systems to absorb changes of state variable, driving variables, and parameters and still persist. [15, p. $17]$

In an attempt to disambiguate resilience, we [6] suggested a generalized definition of resilience for the ecological and socio-ecological domain:

The ability of a system to cope with an external factor that undermines it, with the system bouncing back. [6, p. 121]

This definition was proven to be compatible with definitions given by the Resilience Alliance (c.f. [5]) and by the United Nations International Strategy for Disaster Reduction (c.f. [34, Annex1 p. 6]). To our knowledge, no attempts have been made to model resilience as quality in DOLCE.

Resilience has been linked to vulnerability as both concepts aim to understand socio-ecological systems in face of disturbance.

\subsection{Vulnerability}

Vulnerability can be described according to 27] as the extent to which a natural or social system is susceptible to sustaining damage, for example from climate change. As societies aim to enhance resilience in face of global climate change, the assessment of vulnerability solely, provides a one sided approach to foster adaptation strategies. Consequently, there is a need for the convergence of both theoretical and practical approaches of both concepts [24]. 
The MONITOR ${ }^{3}$ project embarked on the challenge of developing a risk ontology to act as a reference framework for risk management. The concept of vulnerability was modeled and defined as the quality of the objects of an environment, which determines damage, given a defined hazardous event [19].

\subsection{Affordances}

The notion of affordance was introduced by Gibson [1112]. Gibson defined affordances as follows:

The affordance of anything is a specific combination of its substance and its surfaces taken with reference to an animal [11, p. 67]

[A]ffordances are properties of things taken with reference to an observer [12, p. 137]

For example, a chair offers a sitting affordance to a human and a hollow tree has a shelter affordance to a bat or owl. Yet, there exists no definition of affordances that is commonly agreed-upon. Affordances are often seen as either relational qualities, that exist as relation between the environment and the agent (e.g. [32]), or as qualities of (an object in) the environment taken with reference to the agent. The latter view would benefit from an ODP for referential qualities. Definitions supporting the latter view have been given for example by Heft 14, p. 3] and Turvey [33, p. 174].

Today affordances play an important role, not only in their original field of psychology, but they are applied for example in design [25, robotics [31, navigation and way-finding [30] and similarity analysis [17]. However, the potential of affordances is far from exploited. One reason for this is that affordances are challenging to model in formal theories, especially ontologies for the semantic web. The first author has recently suggested to model affordances as qualities in DOLCE [28]. However, in the cases where affordances were modeled in ontology, the reference to the observing agent was not made explicit. This takes away the key idea of an affordance.

\section{An Ontology Design Pattern for Referential Qualities}

In the cases addressed in this paper, we do not need to violate the constraint of a unique host, but we need to extend the quality pattern by a referent entity that is required for the quality. Therefore, qualities like affordance, resilience and vulnerability are not relational and they are not of the kind of non-relational qualities that fit the DOLCE EQQS pattern. They have one host entity, but an additional referent that is necessary for the quality to exist. However, Ellis suggested distinct (extrinsic) qualities that objects have in virtue of outside forces [7, as discussed in [38]]. The notion of force comes very close to the terminology of definitions for vulnerability and resilience, and it also fits the dynamic

\footnotetext{
${ }^{3}$ see http://www.monitor-cadses.org/
} 
and potential nature of affordances. A referential quality could then be called a non-relational extrinsic quality.

The resulting pattern can be described as EQRQS pattern with an endurant (E), a quality $(\mathrm{Q})$, a referent entity $(\mathrm{R})$, a quale $(\mathrm{Q})$ and a quality-space $(\mathrm{S})$. The referent is characterized as playing a special referent-role.

The remainder of the section presents the Ontology Design Pattern for referential qualities. In the classification of the Ontology Pattern Initiative 4 this pattern is a Content Ontology Design Pattern. We do not suggest referential qualities as distinct type of quality to be considered in metaphysics, but intend to give knowledge engineers and domain scientists a practical tool to model the described qualities and to be able to account for qualities that do not solely depend on one or more host entities. For practical reasons the scope of this discussion is mostly limited to DOLCE 23 and its commitments.

In the following the ODP for referential qualities is introduced according to the Content ODP Frame suggested in 8. This frame provides eleven slots to introduce a new Content ODP.

UML Diagram. Figure 2 shows the UML diagram of the referential quality design pattern.

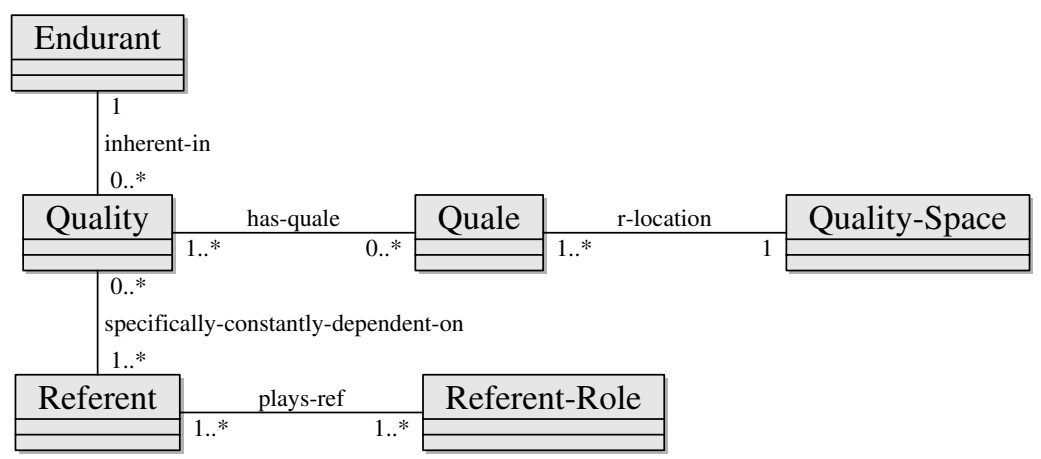

Fig. 2. UML class diagram of the referential quality ODP. The relations are to be read starting from the category quality or from left to right

Generic Use Case (GUC). The referential quality pattern's overall aim is to enable modeling of qualities that have one major host but existentially depend on some external factor.This is a recurring pattern of concepts in Ecology, Economics, Natural Disaster Management, Climate Change, Computer Science, just to name a few. The generic pattern can be phrased as " a quality $Q$ of an entity $X$ taken with reference to another entity $R$ ". These qualities occur in particular when following system-theoretic approaches to model a domain. A system is

${ }^{4}$ see http://ontologydesignpatterns.org 
described not only by its elements but also by relations and interdependencies between these elements [2]. In such systems there often exist qualities that an entity has by virtue of an external factor. For this paper, attention is brought to ecological systems, however, the pattern is not restricted to the domain of ecology, but is kept generic enough to be used in the other aforementioned domains as well.

Local Use Case(s). The local uses cases are equivalent to the examples described in Sect. 3. The referential quality pattern should be capable to account for:

- an affordance as quality of an object taken with reference to an observer.

- resilience as the capacity of a sysem to cope with an external factor.

- vulnerability as the quality of an object with respect to a potential damage.

Affordance, resilience and vulnerability lack a formal ontological definition that makes the concepts operational and allows a consideration of these concepts in and across information systems. Even though conceptual definitions exist, there is a dearth in formal ontological solutions that will allow for semantic integration of information sources and models.

Logic Addressed. The Ontology Design Pattern is modeled in the Unified Modeling Language (UML), it is then encoded in Description Logic and implemented in the Web Ontology Language OWL-DL.

Reference Ontologies. The Ontology Design Pattern uses DOLCE Lit! 5 upper level ontology. As this pattern provides a template to model certain types of qualities, this paper uses especially the DOLCE category qualities. In addition to DOLCE Lite we use the Extended Descriptions and Situations module 6 to specify a role.

Specialized Content ODPs. The ODP for referential qualities is a specialization of the quality ODP in DOLCE. Masolo and Borgo have discussed several approaches to model qualities in formal ontology [22. In DOLCE, a quality depends on one host entity. The quality invokes a quale, i.e. a magnitude of the quality. The quale is located in an abstract quality space. DOLCE uses a framework of entity, quality, quale and quality-space [23]. This ODP extends the DOLCE EQQS pattern with a referent.

Composed Content ODPs. The ODP for referential qualities does not formally compose of other ODPs in its most general form. Nonetheless, the pattern can be seen to compose of the quality pattern that is specialized with a role pattern. Unfortunately, there is no role pattern that is general enough to allow endurants, perdurants or qualities to play the referent role. There exist patterns for special roles, for example the Objectrole7, that can be used to model the role part of the referential quality pattern. Hence, in case the referent-role

\footnotetext{
${ }^{5}$ http://www.loa-cnr.it/ontologies/DOLCE-Lite.owl

${ }^{6}$ http://www.loa-cnr.it/ontologies/ExtendedDnS.owl

7 see http://ontologydesignpatterns.org/wiki/Submissions:Objectrole
} 
is played by a physical-object, the pattern is implemented as composition of the DOLCE quality pattern and the Objectrole pattern.

A special case occurs when the referent is a referential-quality itself, then the pattern can be recursively applied to realize a composition of various referential quality patterns.

Formal Relation. Referential_Quality $(e, q, q l, q s, r, r r)$, where $e$ is an endurant, perdurant or quality, $q$ is a referential quality that inheres in $e, q l$ is a quale of $q$ in the quality space $q s, r$ is an endurant, perdurant or quality that plays a referent-role $r r$ for the referential quality $q$.

\section{Sensitive Axioms.}

Referential_Quality $(e, q, r)={ }_{d f} Q U(q)$

$\wedge(E D(e) \vee P D(e) \vee Q U(e)) \wedge$ inheres $(q, e)$

$\wedge(E D(r) \vee P D(r) \vee Q U(r)) \wedge \quad \wedge(\exists \Phi, s:$ subsumes $(\Theta, \Phi) \wedge \Phi(s))$

$\wedge$ specifically - constantly - dependent - on $(q, r)$

$\wedge$ plays - ref $(r, s)$

where $\Theta$ is the category Role in DOLCE. Note that according to the DOLCE definition of constant specific dependence, $q$ and $r$ can not be equivalent [23, $\operatorname{Dd}(70)$, p. 31].

Explanation. Many existing approaches to model a quality that depends on an external factor lead to unsatisfactory results. The referential quality design pattern suggests a practical approach to model such qualities that is compatible with DOLCE. The DOLCE pattern for qualities comes with qualia and qualityspaces. This already anticipates a future operationalization of these qualities.

OWL(DL) encoding (Manchester syntax, excerpt 8 )

Class: referent-role

SubClassOf:

edns:role

Class: referential-quality

SubClassOf:

dol:quality

and (dol:specifically-constantly-dependent-on

some (rq:plays-ref some rq:referent-role))

\section{Implementation of Affordance, Resilience and Vulnerability}

This section presents the exemplary implementations of vulnerability taken with reference to a hazardous event, resilience taken with reference to an external factor, and an affordances taken with reference to an agent. These examples

\footnotetext{
${ }^{8}$ the complete implementation along with the examples presented in this paper encoded in the OWL Manchester Syntax are available online at http://www.jensortmann.de/ontologies/odp4refprop.html
} 




Fig. 3. UML class diagram of vulnerability in the MONITOR project, modeled according to the referential quality ODP

demonstrate potential applications. However, the ontological choices made to restrict the host and referent of the quality do not necessarily reflect the full scope of the modeled concepts.

The implementation of vulnerability is based on results from the MONITOR project. Hence, it comprises of the referential-quality vulnerability, that is specifically constantly dependent on a damage-potential 9 . The MONITOR project describes damage potential as a quality of the environment. In our implementation we only model the damage potential as quality, but do not model an environment explicitly. From the MONITOR account of vulnerability we concluded that the quale of vulnerability is a defined damage extent, which resides in a special quality space for damage extents. damage-extent is a subcategory of quale. Our implementation does not make the quality space explicit. Figure 3 shows the UML diagram of the vulnerability implementation.

As an example of how to apply vulnerability as a referential quality, we implemented a small set of instances that reflect the vulnerability of a society to an earthquake for the case of Haiti. We introduced a subcategory society of agent, because we treat the society as one whole, abstracting from its constituting institutions, government and people. The category society has one individual called haitian-society. The vulnerability individual is called haitian-vulnerability-to-earthquake and is member of the newly introduced subcategory vulnerability-to-earthquake. The referent-role is played by an individual haitian-damage-potential that we simply introduce as member of quality. The quale of vulnerability is a member of the defined-damage-extent, which is labeled as earthquake-damage-extent. The quale requires further characterization. It could stand for a damage assessed in monetary value or number of injured and dead. The quality space would be modeled accordingly, for example as metric space for money, or as space for counts in the case of victim numbers.

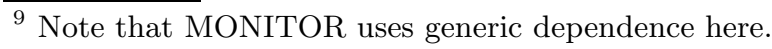




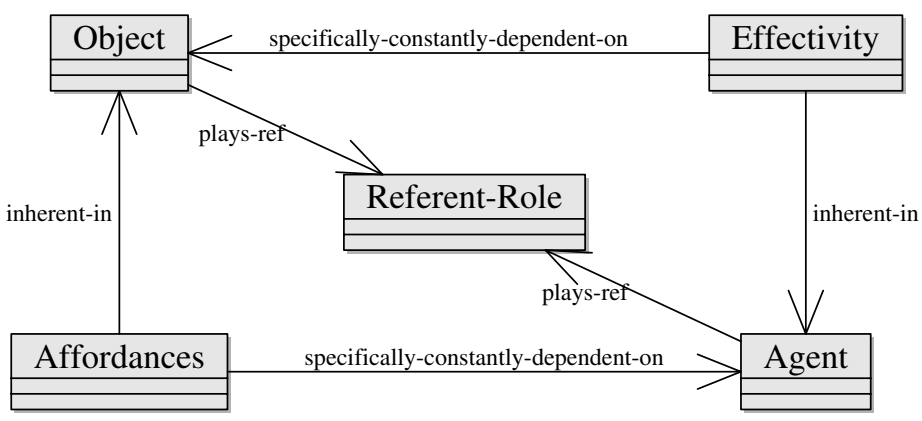

Fig. 4. UML class diagram of an affordance modeled according to the referential quality ODP. For better clarity the cardinalities are not depicted here, but are the same as in Fig. 2 Instead, the associations are directed in this diagram.

The implementation of resilience is based on 6]. It classifies resilience as referential-quality. Aiming for a general account, we leave the external factor as well as the host for resilience unspecified and resort to the category spatio-temporal-particular in DOLCE. We have implemented a specific example and made it available online, further details are left out here.

The implementation of an affordance is based on Turvey's 33] definition of an affordance contrasted with an effectivity. Therefore the implementation comprises two referential-qualities: affordance and effectivity. Both are implemented as direct subcategories of referential-quality. The implementation enforces the referent of an affordance to be the host of an effectivitiy, which in turn needs to be a DOLCE agent. To do justice to Turvey's dispositional nature of affordance, the referent of the effectivity must host the affordance. Figure 4 illustrates the implementation of affordance as UML diagram.

An additional exemplary implementation with individuals of a concrete affordance serves to make the affordances example more graspable and easier to evaluate. One of the most cited investigation of an affordance is Warren's [37. account of the stair-climbing affordance that is provided by a step with a riser height to a person with matching leg-length. The implementation of stair-climbability introduces two new categories for the stair-climbing affordances and for the stair-climbing effectivity. Individuals exist for the physical-object step1 that hosts the referential-quality step1-climbability-affordance and depends on the agent Susan with the effectivity leg-length 10 . Two referent-role individuals fill the respective object-property slots for the individuals of affordance and effectivity. Qualia and quality spaces are not modeled in the stair-climbing example.

$\overline{10}$ The leg length as effectivity is an abstraction that Warren 37. made in his model. 


\section{Evaluation}

There is no strict methodology to evaluate ODPs as yet but only some criteria, such as reusability, to assess the success of an ODP [10]. Therefore, a full evaluation of the suggested ODP cannot be given at this stage. However, this section will shed some light on how well the ODP solved the local use-cases and asses some analytical criteria taken form information systems evaluation.

\subsection{Evaluation of the ODP against the Local Use Cases}

The ontological account of vulnerability is taken from the MONITOR project. It fits the referential quality pattern nearly perfectly. The only difference is that MONITOR suggests a generic dependence between vulnerability and its host, whereas the referential quality pattern requires a constant specific dependence. We chose the specific dependence to express that a particular referential quality depends on a particular referent, but not on a category of referents. Additionally, generic dependence cannot be implemented in OWL-DL, the target language of this ODP. Furthermore, the choice of specific or generic dependence seems to boil down to the philosophical stance on the environment as being shared or being individual. This issue is out of scope of this paper, but both views are supported in the literature. Especially, Turvey's 33] use of singular forms suggests that his objects of discourse are particulars, not universals.

The example of vulnerability to an earthquake could be implemented in DOLCE. Nonetheless, in a real scenario this example has to be integrated with domain ontologies that account for earthquakes. However, the evaluation should rather prioritize the potential for application in a real project like MONITOR. Here we find our pattern is compatible with the ontology suggested in MONITOR, and can lend support to their modeling decisions.

The implementation of resilience as category in DOLCE was straight forward and directly captures the general definition of resilience [6]. A small example showed that it is possible to create individuals that reflect the resilience of a society to sea-level rise. However, the implementations of resilience and also vulnerability do not make these concepts fully operational as yet. The definitions of structured quality spaces are still missing. As soon as qualia and structures are identified in the domain qualia and quality spaces can be defined. Probst [29] suggested a classification of structured quality spaces and an approach to model them in DOLCE.

The challenge in the affordance use case was to model an affordance as property of a thing taken with reference to an observer [12. The ODP gives a direct implementation of this in modeling the affordance as quality of an object that is specifically constantly dependent on an agent. The effectivity is modeled as quality of the agent who plays the referent role. An actual realization of one example of a stair-climbing affordance showed that it is possible and feasible to implement affordances with this ODP in DOLCE and that this implementation complies with a well-established theory of affordances. The affordance example also demonstrates how two referential quality patterns can be composed. 


\subsection{Analytical Evaluation}

In [4, the authors suggested five criteria to evaluate information systems: Significance, internal validity, external validity, confirmability/objectivity and reliability/dependability/auditability. Information system engineering is close enough to ontology engineering and the suggested categories are defined broad enough to use them to evaluate the ODP for referential qualities.

Significance. Referential qualities can be deemed as important building blocks of domain ontologies that account for systems or networks. The whole idea of an ontology design pattern is to turn the theoretical significance of a concept such as referential quality, into a practical significance. The pattern provides a small modular extension to DOLCE that allows to account for these qualities. The authors make clear that the theory of referential qualities is not a contribution to metaphysics, and that the aim is rather to give domain experts a handy template to model common structures in their domain. The practical significance can only be evaluated in terms of the adoption and use of this pattern in the future. The MONITOR project already employs this pattern, without making the pattern itself explicit. The authors own ongoing work on resilience and affordances will also benefit from this pattern.

Internal Validity. The implementation of the three local use cases demonstrates that the ODP works and can be applied in practice. A comparison with relational and non-relational qualities and their respective modeling approaches has revealed the shortcomings of modelling these qualities in formal ontology with respect to requirements stated in the literature on affordances, resilience and vulnerability. The ODP for referential qualities overcomes these shortcomings.

External Validity. The ODP extends an existing pattern for non-relational qualities and uses DOLCE. The implementation of the use-cases conforms with theory in the domain, but rival theories exist that might require a different pattern. The findings in this paper are compatible with for example the specification suggested in the MONITOR project [19] and in Warren's stair climbing experiment [37.

In general, the idea of ODPs and modularity in ontologies is to increase the reusability of ontologies. This pattern is intentionally kept simple to achieve this goal. The nature of ODPs is to make the theory transferable. However, boundaries for the use have not been suggested. The authors see a huge potential for application in a variety of domains that deal with systems and networks in any form. Both authors are currently employing the pattern in their ongoing thesis work.

Confirmability/Objectivity. The design pattern is introduced in a formal way according to 8 . An OWL-DL implementation of the ODP and of the examples is available online. The theory of referential qualities is inspired by ecological systems and the language used by domain scientist in ecology. An ecological bias cannot be ruled out. Furthermore, the commitment to DOLCE and its way of modeling qualities and qualia implies a cognitive bias of the theory. 
Reliability/Dependability/Auditability. The research objective has been made clear in natural language. A detailed description of the OPD is available in formal and natural language. The basic constructs can be easily retrieved from the ODP frame given in Sect. 4, while the implementations are available online.

\section{Conclusion and Future Work}

This paper formally introduced an Ontology Design Pattern for referential qualities. We have discussed the idea of referential qualities and introduced the pattern according to a suggested frame. Three use cases demonstrated the practicability of the pattern. Finally, the paper evaluated the patterns with respect to the use cases together with its general engineering grounds. The pattern is kept general and simple to ensure a flexible and easy application. The pattern has the potential to facilitate ontological modeling of ecological systems. It thereby paves the way for a stronger integration of ecological models and datasets.

Future work will be at first a further application and exploration of the ODP. A promising aspect of referential qualities is their compatibility with Kuhn's Semantic Reference Systems [2021] and Probst's semantic reference system for observations and measurements of qualities 29. This opens the door for the operationalization that the presented concepts still lack. Operationalisation entails the physical and mental measurement of a concept relative to a reference framework [3. The process of operationalization is an important step to a clear understanding of a concept. Finally, one of the reviewers suggested to consider the Description and Situations Ontology [9] as alternative to the comparably strong commitment to DOLCE.

Acknowledgements. The authors gratefully acknowledge the reviewers comments and suggestions. This work has been partly supported through the International Research Training Group on Semantic Integration of Geospatial Information by the DFG (German Research Foundation), GRK 1498.

\section{References}

1. Adger, W.: Social and ecological resilience: are they related? Progress in Human Geography 24(3), 347-363 (2000)

2. von Bertalanffy, L.: An outline of general system theory. The British Journal for the Philosophy of Science 1(2), 134-165 (1950)

3. Bridgeman, P.: The logics of modern physics. Beaufort Books (1927)

4. Burstein, F., Gregor, S.: The systems development or engineering approach to research in information systems: An action research perspective. In: Hope, B., Yoong, P. (eds.) Proceedings of the 10th Australasian Conference on Information Systems, pp. 122-134. Victoria University of Wellington, New Zealand (1999)

5. Carpenter, S., Walker, B., Anderies, J.M., Abel, N.: From Metaphor to Measurement: Resilience of What to What? Ecosystems 4(8), 765-781 (2001)

6. Daniel, D., Ortmann, J.: Disambiguating Resilience. In: Schwering, A., Pebesma, E., Behnke, K. (eds.) Geoinformatik 2011 - Geochange. ifgiprints, vol. 41, pp. 117-125. AKA Verlag, Heidelberg (2011) 
7. Ellis, B.: Scientific Essentialism. Cambridge Studies in Philosophy. Cambridge University Press, Cambridge (2001)

8. Gangemi, A.: Ontology Design Patterns for Semantic Web Content. In: Gil, Y., Motta, E., Benjamins, V., Musen, M. (eds.) ISWC 2005. LNCS, vol. 3729, pp. 262-276. Springer, Heidelberg (2005)

9. Gangemi, A., Mika, P.: Understanding the Semantic web Through Descriptions and Situations. In: Chung, S., Schmidt, D.C. (eds.) CoopIS 2003, DOA 2003, and ODBASE 2003. LNCS, vol. 2888, pp. 689-706. Springer, Heidelberg (2003)

10. Gangemi, A., Presutti, V.: Ontology design patterns. In: Handbook on Ontologies, 2nd edn. International Handbooks on Information Systems, pp. 221-243. Springer, Heidelberg (2009)

11. Gibson, J.: The Theory of Affordances. In: Shaw, R.E., Bransford, J. (eds.) Perceiving, Acting, and Knowing: Toward an Ecological Psychology, pp. 67-82. Lawrence Erlbaum Associates Inc., Hillsdale (1977)

12. Gibson, J.: The ecological approach to visual perception. Lawrence Erlbaum, Hillsdale (1979)

13. Handmer, J.W., Dovers, S.R.: A typology of resilience: Rethinking institutions for sustainable development. Organization \& Environment 9(4), 482-511 (1996)

14. Heft, H.: Affordances and the body: An intentional analysis of Gibson's ecological approach to visual perception. Journal for the Theory of Social Behaviour 19(1), 1-30 (1989)

15. Holling, C.: Resilience and stability of ecological systems. Annual Review of Ecology and Systematics 4, 1-23 (1973)

16. ICSU: Earth system science for global sustainability: The grand challenges. Tech. rep., International Council for Science, Paris (2010)

17. Janowicz, K., Raubal, M.: Affordance-Based Similarity Measurement for Entity Types. In: Winter, S., Duckham, M., Kulik, L., Kuipers, B. (eds.) COSIT 2007. LNCS, vol. 4736, pp. 133-151. Springer, Heidelberg (2007)

18. Klein, R., Nicholls, J., Thomalla, F.: Resilience to natural hazards: How useful is this concept? Global Environmental Change Part B: Environmental Hazards $5(1-2), 35-45$ (2003)

19. Kollartis, S., Wergels, N.: MONITOR - an ontological basis for risk managment. Tech. rep., MONITOR (January 2009)

20. Kuhn, W.: Semantic reference systems. International Journal of Geographical Information Science 17(5), 405-409 (2003)

21. Kuhn, W., Raubal, M.: Implementing Semantic Reference Systems. In: Gould, M., Laurini, R., Coulondre, S. (eds.) Proceedings of the AGILE 2003 - 6th AGILE Conference on Geographic Information Science, Lyon, France, pp. 63-72. Presses polytechniques et universitaires romandes, Lausanne (2003)

22. Masolo, C., Borgo, S.: Qualities in formal ontology. In: Foundational Aspects of Ontologies (FOnt 2005) Workshop at KI, pp. 2-16 (2005)

23. Masolo, C., Borgo, S., Gangemi, A., Guarino, N., Oltramari, A.: Wonderweb deliverable D18 ontology library (final). ICT Project 33052 (2003)

24. Miller, F., Osbahr, H., Boyd, E., Thomalla, F., Bharwani, S., Ziervogel, G., Walker, B., Birkmann, J., van der Leeuw, S., Rockstrôm, J.: Resilience and vulnerability: Complementary or conflicting concepts? Ecology and Society 15(3) (2010)

25. Norman, D.: The Design of Everyday Things (Originally published: The psychology of everyday things). Basic Books, New York (1988)

26. Noy, N., Rector, A.: Defining N-ary Relations on the Semantic Web. Working group note, W3C (April 2006), http://www.w3.org/TR/swbp-n-aryRelations/ 
27. Olmos, S.: Vulnerability and adaptation to climate change: Concepts, issues, assessment methods. Tech. rep., Climate Change Knowledge Network, CCKN (2001)

28. Ortmann, J., Kuhn, W.: Affordances as Qualities. In: Galton, A., Mizoguchi, R. (eds.) Formal Ontology in Information Systems Proceedings of the Sixth International Conference (FOIS 2010). Frontiers in Artificial Intelligence and Applications, vol. 209, pp. 117-130. IOS Press, Amsterdam (2010)

29. Probst, F.: Observations, measurements and semantic reference spaces. Applied Ontology 3(1-2), 63-89 (2008)

30. Raubal, M.: Ontology and epistemology for agent-based wayfinding simulation. International Journal of Geographical Information Science 15(7), 653-665 (2001)

31. Rome, E., Hertzberg, J., Dorffner, G.: Towards Affordance-Based Robot Control: International Seminar Dagstuhl Castle, Germany. LNCS. Springer, Heidelberg (June 2006) (revised papers)

32. Stoffregen, T.A.: Affordances as properties of the animal-environment system. Ecological Psychology 15(2), 115-134 (2003)

33. Turvey, M.: Affordances and prospective control: An outline of the ontology. Ecological Psychology 4(3), 173-187 (1992)

34. UN/ISDR: Living with risk: A global review of disaster reduction initiatives. Tech. rep., Inter-Agency Secretariat of the International Strategy for Disaster Reduction (2004)

35. Walker, B., Carpenter, S., Anderies, J., Abel, N., Cumming, G., Janssen, M., Lebel, L., Norberg, J., Peterson, G., Prichard, R.: Resilience Management in Socialecological System: a Working Hypothesis for a Participatory Approach. Conservation Ecology 6(1) (2002)

36. Waller, M.A.: Resilience in ecosystemic context: evolution of the concept. Am. J. Orthopsychiatry 71(3), 290-297 (2001)

37. Warren, W.: Perceiving affordances: Visual guidance of stair climbing. J. of Experimental Psychology: Human Perception and Performance 10(5), 683-703 (1984)

38. Weatherson, B.: Intrinsic vs. Extrinsic Properties. In: The Stanford Encyclopedia of Philosophy, Fall 2006 edn. (2006),

http://plato.stanford.edu/archives/fall2008/entries/

intrinsic-extrinsic/ 